Samuel N., aged 30 , a porter, was admitted under Dr. Fuller's care on May 5. He was complaining of pain in the shoulder and at the epigastrium, apparently in a fixed spot. There was some tenderness on pressure there, and the pain was worse after taking food. He complained of not being able to swallow anything without washing it down with drink. These symptoms had existed for about three months. The day before his admission he had vomited half-a-pint of blood. There was no aneurismal bruit in the chest. 'The painful spot was covered with a sinapism; the bowels, which had been very costive, were opened with senna, and then a mixture containing dilute acid with sulphate of magnesia and infusion of roses was given every six hours. Some blood, but no very great quantity, was brought up during the night. Gallic acid and opium were given the next night, and repeated two or three times during May $8 \mathrm{th}$, in addition to a turpentine mixture. About four P.M. on the 8th, he threw up more than half-a-pint of blood, and soon sank from suffocation, as it appeared, caused by inability to dislodge more of it.

On post mortem examination the body was seen to be in good condition. There was a large perforation in the osophagus close to the bifurcation of the trachea, with smooth rounded edges, partly filled up by clot, which appeared of old standing. The œsophagus itself was otherwise healthy. This opening was immediately opposite to an equally large opening in the arch of the aorta, just below the exit of its three branches. A small sac had been formed in the cellular tissue between the two tubes, not much larger than a chesnut; also filled with loose clot. The left bronchus passed through this, but no communication was formed with the air tube. The heart was healthy. The aorta was studded with atheromatous deposit and the inner coat was elevated in many places and thickened. Here and there small patches of fibrine projected from the lining mernbrane of the artery. Just below the opening of the aneurism, a space existed, in which the inner, and apparently part of the middle coat of the vessel had been removed, leav ing the yellow elastic fibres bare. The trachea and bronchi were stained with blood, and numerous extravasations existed in many parts of the left lung, but (as already stated) no com. munication was formed with any part of the air tube. The stomach was distended by an enormous soft clot of blood. It was otherwise healthy.

\section{OBtiginal Commumations.}

\section{ON DISEASES OF JOINTS.}

By Holmes Coote, Esq., F.R.S.C., Assistant-Surgeon to St. Bartholomew's Hospital, and to the Royal Orthopædic Hospital, etc.

\section{THE ANKLE-JOINT.} [Continued from page 349.]

THe deformity called talipes valgus, or flat foot, must be distinguished from simple sinking of the inner ankle and eversion of the foot, consequent upon yielding of the internal lateral ligament. 'The arch of the foot, which in the former affection is lost, is here retained, and the only treatment usually necessary consists in the application of a boot with outside iron upon which straps act to draw the weakened ankle to its proper shape. In course of time, parts acquire sufficient firmress for the discortinuance of mechanical support.

Talipes valgus, or flat foot, involves a much more serious amount of deformity, and is attended with great stiffness and rigidity of the limb. The arch of the foot is lost in the antero. posterior direction, owing to the sinking of the astragalus from the cup-like cavity formed by the os calcis, the calcaneoscaphoid ligament, and the os scaphoides, into which the head of the bone is in the normal state received; and in the transverse direction, owing to the yielding and stretching of the ligaments connecting the scaphoid, cuboid, and cuneiform bones. The lateral ligaments are affected secondarily, so that the external malleolus becomes approximated to the outer side of the os calcis.

The amount of pain and inconvenience which patients thus afflicted suffer is far greater thar commonly supposed. It is true, they may in some instances manage to shuffle many miles over the smooth paved streets of London, but they have quite lost the elastic spring, or the adaptive power of the foot, by which one is enabled to travel over uneven and rugged places, or to save oneself from falling. But the sinking of the arch of the foot is in itself a very painful process. Patients cannot stand long at a time, and they bitterly complain of a constant aching just in the situation of the elastic calcaneo. scaphoid ligament.

In December 1848, I saw a case in St. Bartholomew's Hospital, under the care of one of my colleagues, of a lad, the subject of talipes valgus, who, falling from a considerable height upon the feet, fractured the right astragalus. The case did well, under proper treatment; but the nature of the accident illustrated the fact, that the weight of the body would be transmitted directly by the tibia to this bone of the tarsus, as it came to the ground.

In flat foot, the muscles are often in a remarkable state of spasm. I saw a little girl, who might have seemed to a casual observer to be suffering from talipes varus, for she walked on the outer side of the feet. When put on a sofa, however, the muscles became quieter, and the real nature of the deformity was apparent. 'The explanation of the case was easy. The child suffered constant pain from the sinking of the astragalus whenever she stood upright; for the weight of the body pressed on the elastic calcaneo-scaphoid ligament. To rectify this, the anterior and posterior tibial muscles were thrown into a state of spasm, by which the inner border of the foot was raised, while the flexor muscles produced antero-posterior contraction of the tarsus, with flexing of the toes. The same condition of general spasms is often seen when the two peronei muscles are morbidly tense. Rest of the limb becornes important as an element of diagnosis ; for, by quieting the muscles generally, those tendons which are permanently tense become easily distinguished from the rest.

W. R. Adams, in the Dublin Journal of Medical Science, vol. xix, 18t1, relates the particulars of a case of flat foot in a female aged 60 , dissected by Mr. Smith. The ankle and the tarsal joints were remarkably stiff and rigid; and, upon the removal of the soft parts, bony vegetations were seen springing from the articular margins of all the tarsal bones. The direction of the long axis of the os calcis was more horizontal than natural ; but the astragalus, the os scaphoides, and the calcaneoscaphoid ligament, were changed more than any other structure in the foot. The long axis of the astragalus was horizontal; from the upper surface of this bone arose an osseous growth, which exteuded half an inch in front of the fibula, so that the ankle joint was nearly as immoveable as by osseous anchylosis. This immobility of the ankle appeared to be compensated for by increased mobility of the tarsal joints. The head of the astragalus was large, and the concavity formed for its reception by the os scaphoides was greatly enlarged. The calcaneoscaphoid ligament, which in its normal state supports the under surface of the head and the neck of the astragalus, was in this case ossified and converted into a large crescentic bone (a new sesamoid bone), nearly an inch in its long diameter, serving by its under surface for attachment to the tibialis posticus, and by its upper surface forming, with the concavity of the os scaphoides, a large articular surface, as big as and of similar shape to the glenoid cavity of the scapula. The narrowest point of the ovoid was directed downwards inwards, and this corresponded with the remarkable prominence on the inner side of the sole of the foot noticed in all cases of flat foot. The other bones exhibited no abnormity, excepting the numerous exostoses at the line of separation of the differen bones.

The tendons which require division in severe cases of this deformity are those of the peronei and the extensor communis digitorum. "In congenital cases," observes Mr. Tamplin, " as far as my observation goes, the measure is invariably necessary, although the deformity may be slight, and the foot is easily brought into and held in position." The moment the feet are released from the instrument, the deformity-returns, even after a period of twelve to eighteen months. But subsequent division of the peronei and the common extensor of the toes is speedily followed by a cure of the deformity, and removal of all tendency to contraction.

In severe congenital cases, when the deformity has been al lowed to exist for years without any attempt being made to relieve it, every muscle surrounding the joints is contracted. The following case is interesting. "A patient, fifteen years of age, was born with this deformity in both feet, and about two years since, was operated upon and treated mechanically, without relief. The patient was wholly unable to walk from the great pain occasioned when the feet were subjected to the weight of the body. Upon attempting to adduct the feet, the peronei and common extensor were rendered ex- 
tremely tense; upon attempting to flex the foot, the tendo Achillis was also tense; and, upon an attempt to depress them, the anterior tibial and extensor proprius pollicis were also tense, there being the smallest possible amount of motion in the joint, and that of an unyielding character. I therefore proposed the division of the tendons of the whole of these muscles, as the only prospect of relief, which was assented to. I first divided the peroneus longus and brevis; then the extensor communis digitorum and peroneus tertius ; afterwards, the anterior tibial and extensor proprius pollicis; and lastly, the tendo Achillis. Lint and bandages were applied, and allowed to remain on a week, the patient having suffered no pain beyond that immediately following the operation. At the end of that time, I applied one of Scarpa's shoes, and flexed and extended the foot alternately night and morning, so that the uniting medium of each tendon might be acted upon, the foot being adducted the whole time. At the end of three weeks, the motion of the joint was restored perfectly, and the patient possessed the power of flexing and extending it at will." (Tamplin, On Deformities, p. 77.)

The mechanical treatment of talipes valgus consists in gradually adducting and depressing the foot at the same time that the arch is supported, so that the new uniting medium becomes stretched while the bones of the foot forming the arch are pressed into the normal position. The usual practice at the Royal Orthopædic Hospital is to apply first a splint reach ing from below the knee to the inner ankle, with a spring attached to its extremity, to extend the length of the foot; the spring having a soft pad attached directly opposite and rather beneath the os scaphoides. By this instrument, the arch of the foot may, with proper bandaging, be restored, with great comfort to the feelings of the patient. The arch of the foot having once been raised, the flexion of the foot may be commenced by Scarpa's shoe. Finally, the patient should be supplied with a boot with an outside iron support, and a pad in the interior corresponding with the arch of the foot, that the patient may take exercise with safety, at the same time that the ligaments are gaining strength.

[To be continued.]

\section{APPLICATION OF BELLADONNA AS AN ANTILACTESCENT.}

By William Newaran, Esq., Fulbeck, near Grantham.

Several papers have appeared within the last two years in the columns of the Britrsh Medical Journal, advocating the employment of belladonna in cases where lacteal abscess is threatened; and also in instances where other causes render it advisable to check any further secretion of milk.

In one of these cases, supplied by Mr. J. Burrows, in the JourNal of March 28th, 1857, the belladonna was used in conjunction with other remedies; and the value of the case, as a proof of the efficacy of the drug, is thereby impaired. Another mention from the Westminster Hospital, in the Journal of April 4th, 1857, refers simply to its use in threatened lacteal abscess. I am not aware that any notice exists defining the separate classes of cases in which the remedy may be available; and this deficiency I would attempt, though most imperfectly, to supply.

I have had reason to regard belladonna as an antilactescent, and have resorted to it as such.

1. In cases where suppression of the secretion is advisable; e.g.,

(a) Where the child has been stillborn, or has died in the first few days after birth.

(b) Where it is desirable to wean the child even suddenly, the flow of milk still remaining unabated.

2. In cases where engorgement of the gland has occurred, and lacteal abscess is threatening; e.g.,

(a) Where, from existence of flat nipples, injury to the breast from prior abscess, or from deficient secretion of milk, the mother is, after some short and futile attempt at suckling, obliged to desist; here the gland, though not called into prolonged exercise, is yet excited, and its lia. bility to inflammation thereby increased.

(b) Where the mother has suckled, and is continuing to suckle her child: but in whom, from some accidental cause or other, congestion of the breast has been set up.

I have resorted to belladonna in more than a dozen casescomprising instances of each one of the classes I have so hastily sketched, and have not been disappointed in any of them. The cessation of the secretion, or the resolution of the engorgement, has very shortly followed the application. The drug has been solely trusted in; and I have not in any case given the routine doses of calomel, salts, etc., often resorted to in similar instances; indeed, no medicine whatever was exhibited.

I have employed the extract softened with nearly an equal part of glycerine; and have applied this in a ring round the breast external to the areola. It has rarely been required for more than twenty-four hours.

How far it may be possible to check the secretion of milk in one breast on account of threatened abscess, allowing the child at the same time to continue sucking the other one, I am quite uncertain. No case of the kind has as yet occurred to me; but I should fear the influence of the belladonna upon the child. No inconvenience has resulted to the mother in the above cases, save that in one instance there was dilatation of the pupils with intolerance of light for a short time.

I would simply draw attention, in closing this short notice, to the great comfort and advantage of supporting the breast in any of the states I have referred to. A handkerchief placed under the gland, with its ends tied round the neck, appears to have more in its favour than the employment of strips of bandage or of adhesive plaster.

\section{Tramsactrons of âdramellyes.}

\section{BATH AND BRISTOL BRANCH.}

ON ULCERATION OF THE OS UTERT.

By J. G. Swayne, M.D., Physician-Accoucheur to the Bristol General Hospital, and Lecturer on Midwifery at the Bristol Medical School.

$$
\text { [Read April 29th.] }
$$

For some years past, the subject of ulceration of the os uteri has received much attention, and has excited no small amount of acrimonious discussion amongst those who especially study diseases of the uterine system. Nevertheless, most contradictory opinions are still held as to the frequency and importance of this ailment; and, as individual experience of any kind is useful in testing the relative value of those opinions, I propose giving the results of my own observations in a certain number of cases, exhibiting illustrations of the best marked instances. The cases have been chiefly collected and the drawings made, during the last two years, from patients under my care at the Bristol General Hospital, and will show in some measure the frequency of simple ulceration of the os uteri, as compared with other organic diseases of that organ. Out of 120 cases of organic uterine diseases, which it was necessary to examine by the touch and speculum, there were 31 of simple non-maiignant inflammation and ulceration of the os uteri; in 23 of these, there was distinct and well marked ulceration; in the remaining 9 , there were merely superficial inflammation and enlargement, or an inflamed and granular condition of the mucous membrane of the part. In 57, or nearly half of the 120 cases, the uterus was prolapsed, or otherwise displaced; in 9 , there were fibrous tumours, polypi, and other non-malignant growths; in 7 , there was carcinoma; and in 16 , various other affections not belonging to either of these classes. Hence we find that there was simple non-malignant ulceration of the os uteri in 2:3 out of 120 cases, or rather more than one-sixth of the whole; thus showing that this affection is, next to displacement of the uterus, the most frequent of the organic diseases. However, the disease would not appear, from my own statistics, to be so common as it would from those of Dr. Bennet, who found 222 cases of uterine ulceration in 300 which he exexamined-i.e., nearly three-quarters of the whole; or even from those of Dr. West, who found 125 cases in $268-i$. e. nearly half. Yet, on the other hand, some practitioners of great experience and acuteness of observation affirm that they have never, either in the living or dead body, seen ulceration of the os and cervix uteri, except of a specific character, such as syphilitic, scrofulous, or cancerous.

The only way in which we can account for so great a discrepancy of opinion as to a matter of fact, is by supposing that observers of so opposite a character attach a different meaning to the term " ulceration". Such appears to be the case. The lesions which are considered to be ulcers by one class of observers are so superficial in character that they are not con- 